\title{
Envejecimiento y propiedades eléctricas de materiales basados en Y-TZP
}

\author{
I. CACHADIÑA, J.D. SOLIER, A. DOMÍNGUEZ-RODRÍGUEZ* \\ Dpto. de Física, Universidad de Extremadura, Avda. de Elvas s/n, 06081 Badajoz \\ *Dpto. de Física de la Materia Condensada, Universidad de Sevilla, 41080 Sevilla
}

Se ha estudiado la respuesta eléctrica de policristales tetragonales de $\mathrm{ZrO}_{2}-3 m o l \% \mathrm{Y}_{2} \mathrm{O}_{3}(\mathrm{Y}-\mathrm{TZP})$ con distintos tamaños de grano, en el rango de baja temperatura $\left(\mathrm{T}<350^{\circ} \mathrm{C}\right)$, para analizar su comportamiento en el proceso de envejecimiento que sufren estos cristales. El espectro de impedancia, que inicialmente muestra dos picos relacionados con las contribuciones de grano y frontera de grano (altas y bajas frecuencias, respectivamente), ha sido ajustado mediante un modelo circuital para analizar sus contribuciones. La evolución de los parámetros de ajuste con los ciclos térmicos ha sido analizada para ver cual es la influencia del proceso de envejecimiento sobre éstos.

Palabras clave: Y-TZP, policristales, propiedades eléctricas, envejecimiento.

Ageing and electrical properties of Y-TZP based materials

The electrical response of $3 \mathrm{~mol} \%$ yttria tetragonal zirconia polycrystals (Y-TZP) was measured as a function of the grain size in the low temperature range $\left(\mathrm{T}<350^{\circ} \mathrm{C}\right)$. The impedance spectrum shows two peaks related to grain and grain boundary effects (high and low frequencies, respectively) that were fitted by a circuit model in order to analyse their respective contributions. The evolution of the fitting parameters with the thermal cycles was also studied to determine the thermal unstability (aging).

Keywords: Y-TZP, polycrystals, electrical properties, aging.

\section{INTRODUCCIÓN}

El interés tecnológico en los policristales tetragonales de circona dopada con itria (Y-TZP) reside, por una parte, en su dureza y/o resistencia a la fractura, la cual es atribuida a la transformación, inducida por tensiones, de los granos de la fase tetragonal a monoclínica $(t \rightarrow m)$; y por otra parte en su alta conductividad eléctrica, fruto de la difusión de vacantes de oxígeno, que los hace interesantes en aplicaciones como sensores de oxígeno, células de combustible, etc.

No obstante, estos materiales presentan la gran desventaja de que en su uso a bajas temperaturas $\left(\sim 250^{\circ} \mathrm{C}\right)$ sus propiedades mecánicas empeoran dramáticamente. Esta degradación tiene su origen en la transformación espontánea $(t \rightarrow m)$ que es acompañada por la formación de macro y microgrietas. Está demostrado que la velocidad de la transformación aumenta con el tamaño de grano y con la presencia de agua o vapor de agua (1-5).

Desde el punto de vista de las propiedades eléctricas, el proceso de envejecimiento, que implica la formación de grietas, debe estar asociado a un cambio en estas propiedades de manera que se produzca una disminución dramática de la conductividad eléctrica. En este trabajo analizamos la dependencia de las propiedades eléctricas con el tamaño de grano y el envejecimiento.

\section{PARTE EXPERIMENTAL}

Las muestras se fabricaron a partir de polvos de $3 \mathrm{~mol} \% \mathrm{Y}$ TZP, suministrados por Tosoh Co. (Japón), que fueron prensa- dos en frío (CIP) a $137 \mathrm{MPa}$ y posteriormente sinterizados a $1400^{\circ} \mathrm{C}$ durante una hora, obteniéndose un material totalmente denso. El tamaño de grano medio de las muestras, determinado por SEM, fue de $0.3 \mu \mathrm{m}$. Para obtener muestras con tamaños de grano mayor se hicieron recocidos a $1550^{\circ} \mathrm{C}$ durante 24 horas y a $1600^{\circ} \mathrm{C}$ durante 10 horas, obteniéndose muestras con tamaño de grano de 0.7 y $1.2 \mu \mathrm{m}$, respectivamente. Una vez cortadas en láminas, se evaporaron electrodos de oro y se midió la repuesta eléctrica en el rango de $20 \mathrm{~Hz}$ a $1 \mathrm{MHz}$ utilizando un Hewlett-Packard HP4284A, realizando diez barridos en frecuencia por cada temperatura. La temperatura varió en pasos de $5^{\circ} \mathrm{C}$ en el intervalo de 150 a $350^{\circ} \mathrm{C}$, y se efectuaron varios ciclos térmicos (calentamiento y enfriamiento) consecutivos. En todos los casos la fluctuación de la temperatura en el proceso de medida eléctrica fue inferior a $1^{\circ} \mathrm{C}$.

\section{RESULTADOS Y DISCUSIÓN}

Es bien conocido que en el espectro de impedancia de las muestras policristalinas hay tres contribuciones: la primera está asociada a la interfase electrodo-muestra y puede dominar en las frecuencias más bajas; subiendo en frecuencia encontramos la contribución de la frontera de grano, y por último a frecuencias altas tenemos la del grano (6-7). Para ajustar este comportamiento experimental se ha utilizado el modelo circuital de la figura 1. En la contribución del volumen la respuesta del grano ha sido modelada con un RC paralelo, donde la resistencia $\mathrm{R}$, tiene en cuenta la contribución de 


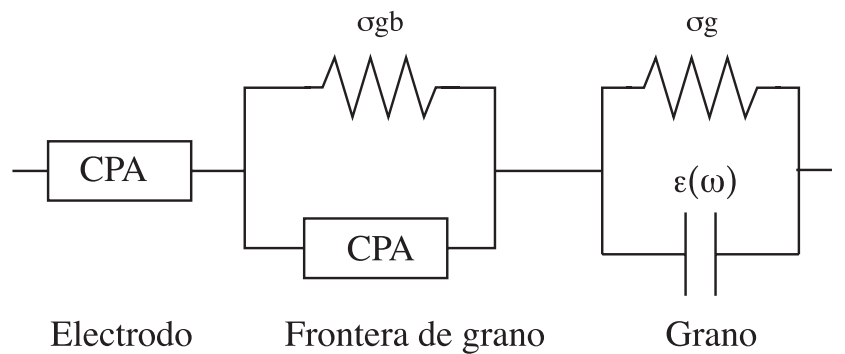

Figura 1. Modelo circuital utilizado para el ajuste de los datos experimentales. La permitividad dieléctrica depende de la frecuencia como la transformada de Fourier de una función de KohlrauschWilliams-Watts.

la conducción iónica, y la capacidad dependiente de la frecuencia angular $(\omega)$ ajusta la dispersión dieléctrica considerando una función respuesta escalón de KohlrauschWilliams-Watts. Este modelo se ha demostrado muy eficaz para ajustar la respuesta eléctrica tanto de monocristales $(8,9)$ como la contribución del grano en policristales $(10,11)$. La frontera de grano está representada por un arco de impedancia (12), formado por la asociación en paralelo de un elemento CPA $\left(z(\omega)=(j \omega \tau)^{-n}\right)$ y una resistencia, y finalmente, la contribución de la interfase electrodo-muestra ha sido modelada como otro CPA.

Los parámetros de ajuste han sido obtenidos por el método de mínimos cuadrados no lineal de Levenberg-Marquardt (12), donde como pesos de ajuste se han utilizado los datos de la precisión del medidor, dados por el fabricante para cada valor de frecuencia e impedancia (11). En la figura 2a se han representado los datos experimentales de la muestra de 0.3 $\mu \mathrm{m}$ a la temperatura de $300^{\circ} \mathrm{C}$ junto con el ajuste de los puntos experimentales y los residuos del ajuste (fig $2 b$ ). Puede verse que el modelo circuital utilizado es válido para representar el comportamiento experimental en todo el rango de frecuencias. Asimismo, están dibujadas las contribuciones del grano (dominante a frecuencias mayores de $4 \times 10^{4} \mathrm{~Hz}$ ) y la frontera de grano (dominante a frecuencias entre $10^{2}$ y $10^{4} \mathrm{~Hz}$ ). La presencia del electrodo puede ser observada en frecuencias menores de $10^{2} \mathrm{~Hz}$ pero sus efectos se extienden significativamente hasta la frecuencia de $10^{3} \mathrm{~Hz}$. Puede verse también que hay zonas del espectro en la que se superponen los efectos, de manera que el ajuste numérico es una herramienta muy útil para separar las diferentes contribuciones. De esta forma obtenemos las conductividades de grano $\sigma_{g}$ y frontera de grano $\sigma_{\mathrm{gb}}$ para cada temperatura para las muestras con diferente tamaño de grano. En las figuras $3 a$ y $3 b$ se han dibujado los diagramas de Arrhenius de la conductividad eléctrica del grano y la frontera de grano para todas las muestras en el primer ciclo térmico. Puede observarse (Fig. 3a) que la conductividad de grano es similar en todas las muestras, mientras que la conductividad de frontera de grano (Fig. 3b) es inferior en las muestras con tamaño de grano menor. En otras palabras, un tamaño de grano menor implica que un portador de carga debe atravesar más fronteras de grano en su movimiento por el policristal.

Se han representado también en la figura 3 los ajustes de tipo Arrhenius en el rango $215^{\circ} \mathrm{C}$ a $300^{\circ} \mathrm{C}$, en los que se han obtenido las energías activación de 1.07, 1.11 y 1.15 eV para las fronteras de grano y $0.92,0.95$ y $0.90 \mathrm{eV}$ para el grano, correspondientes a los tamaños de $0.3,0.7$ y $1.2 \mu \mathrm{m}$, respectivamente. Los valores de energía de activación para el grano
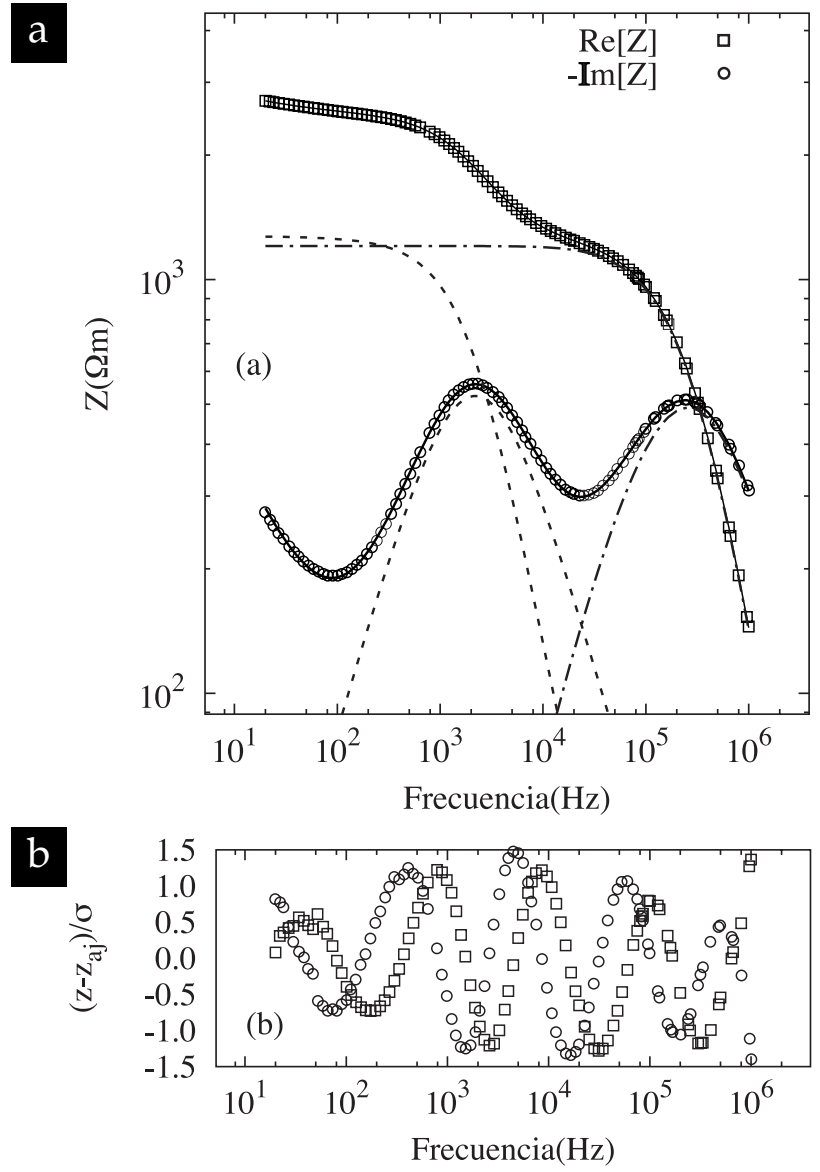

Figura 2. Datos experimentales de la muestra de $0.3 \mu \mathrm{m}$ a $300^{\circ} \mathrm{C}$ con el ajuste de los datos (__ (a) y los residuos de ajuste (b). Se muestran también las contribuciones del grano (._._. y la contribución de la frontera de grano (- - -).

están de acuerdo con los valores de $0.95 \mathrm{eV}$ y $0.92 \mathrm{eV}$ dados por Sellars y Steele (14) y por Badwal (6), para muestras de la misma concentración. También se obtiene un buen acuerdo con los valores de la frontera de grano dados por Badwal (6) de 1.08 y $1.11 \mathrm{eV}$.

Con relación al proceso de envejecimiento de las muestras, el primer efecto puede verse en torno a $300^{\circ} \mathrm{C}$ para tamaños de grano de $1.2 \mu \mathrm{m}$, donde el valor de la conductividad cae abruptamente, sugiriendo la presencia de grietas (mayor resistividad). Tal y como ha sido mostrado por Li y col. (4), con el aumento de tamaño de grano decrece la estabilidad intrínseca de los granos tetragonales, de forma que la muestra $1.2 \mu \mathrm{m}$ debería ser la muestra más inestable de las estudiadas, tal y como puede verse en la figura. Por otra parte, podemos ver cómo cambia el aspecto del espectro de impedancia cuando empieza la rotura. Aprovechando que para la misma temperatura se han realizado 10 barridos en frecuencia consecutivos, se han dibujado en la figura 4 la parte imaginaria de los barridos 9 y 10 de la medida a $305^{\circ} \mathrm{C}$. Los datos del barrido 9 se han tomado desde $1 \mathrm{MHz}$ a $20 \mathrm{~Hz}$ mientras que los del $10^{\circ}$ se han tomado desde $20 \mathrm{~Hz}$ a $1 \mathrm{MHz}$. Puede verse que en la frecuencia de $27 \mathrm{~Hz}$ hay un aumento súbito en la impedancia del barrido 9 al 10 que no afecta a la forma del espectro: éste sigue teniendo los dos picos (asociados al grano y a la fronte- 
a

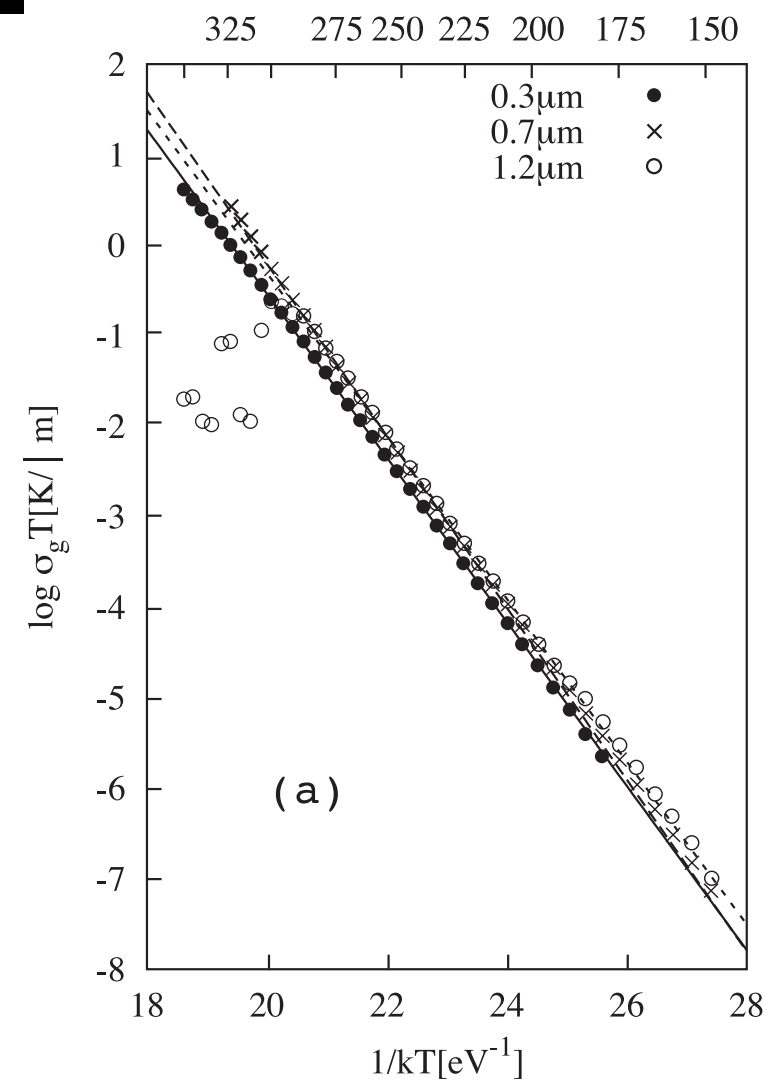

b

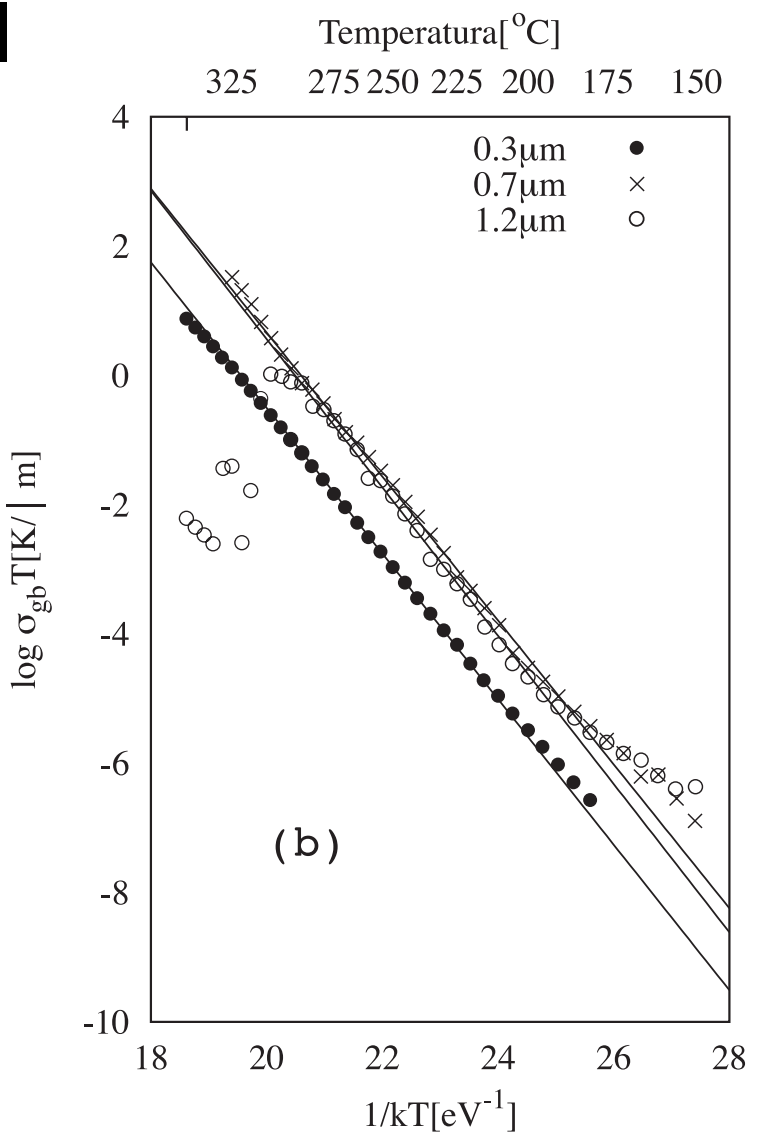

Figura 3. Diagramas de Arrhenius de la conductividad del grano (a) y de la frontera de grano (b) para todas las muestras estudiadas en el primer ciclo térmico.

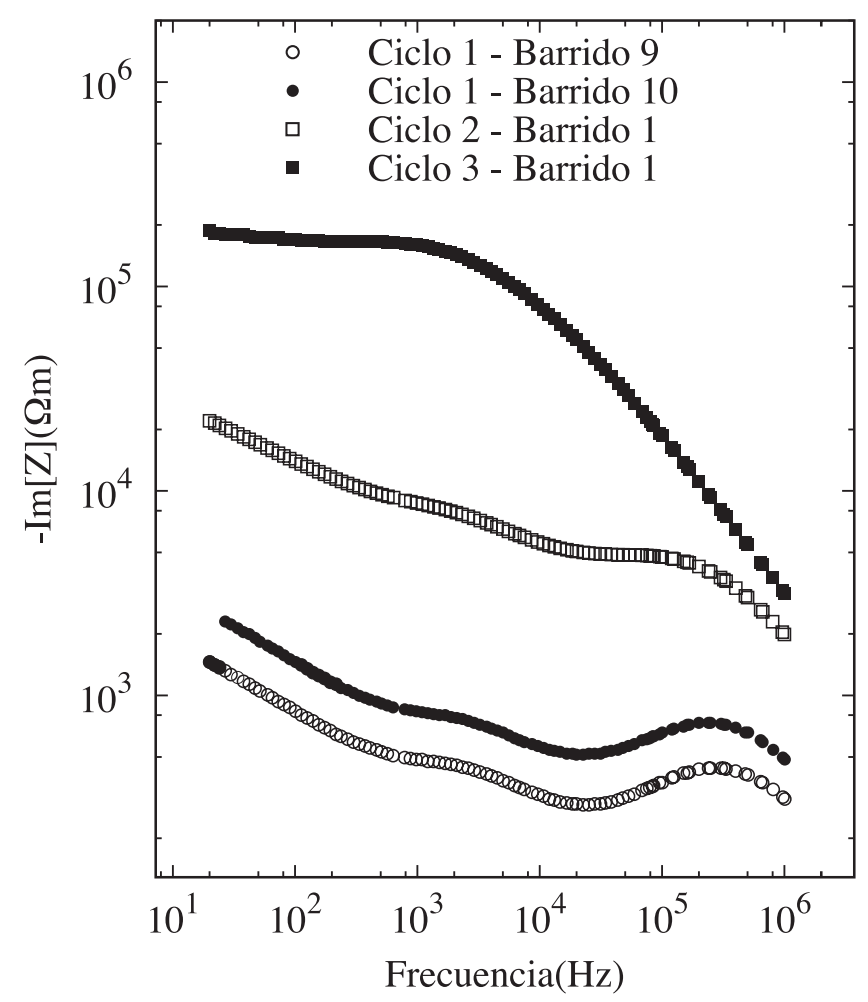

Figura 4. Parte imaginaria de los espectros de impedancia a $305^{\circ} \mathrm{C}$ de la muestra de $1.2 \mu \mathrm{m}$ durante los tres primeros ciclos térmicos.

ra de grano) pero multiplicados por un factor 1.70. Este cambio tan brusco creemos que es debido a la formación de una macro grieta que deja aislada una parte de la muestra, que ya no puede ser utilizada por los portadores de carga, con lo que aumenta la impedancia. Al no haber cambio en la forma del espectro, la función de ajuste sigue siendo válida para representar el comportamiento experimental, sin embargo, los valores de las conductividades y permitividades dieléctricas decrecen abruptamente de acuerdo con este aumento de impedancia aunque la posición del máximo de la parte imaginaria (relacionado con el cociente de permitividad dieléctrica y conductividad) no cambia, lo que nos indica que los mecanismos que contribuyen al espectro de impedancia en este rango de frecuencia son los mismos. En el segundo ciclo térmico el espectro aún recuerda al del primero, sin embargo la caída que hay a la izquierda del máximo de alta frecuencia desaparece. No hace falta esperar mucho para que desaparezca cualquier vestigio de la forma original del espectro; en el tercer ciclo ya no hay ningún rastro de los dos picos que lo formaban en un inicio: la parte imaginaria, queda reducida a un "plateau" a bajas frecuencias seguida de una zona en la que decrece como $\omega^{-0.73}$. Una vez finalizadas las experiencias y retirar la muestra del horno pueden verse a simple vista las fisuras y una evidente falta de cohesión. 
a

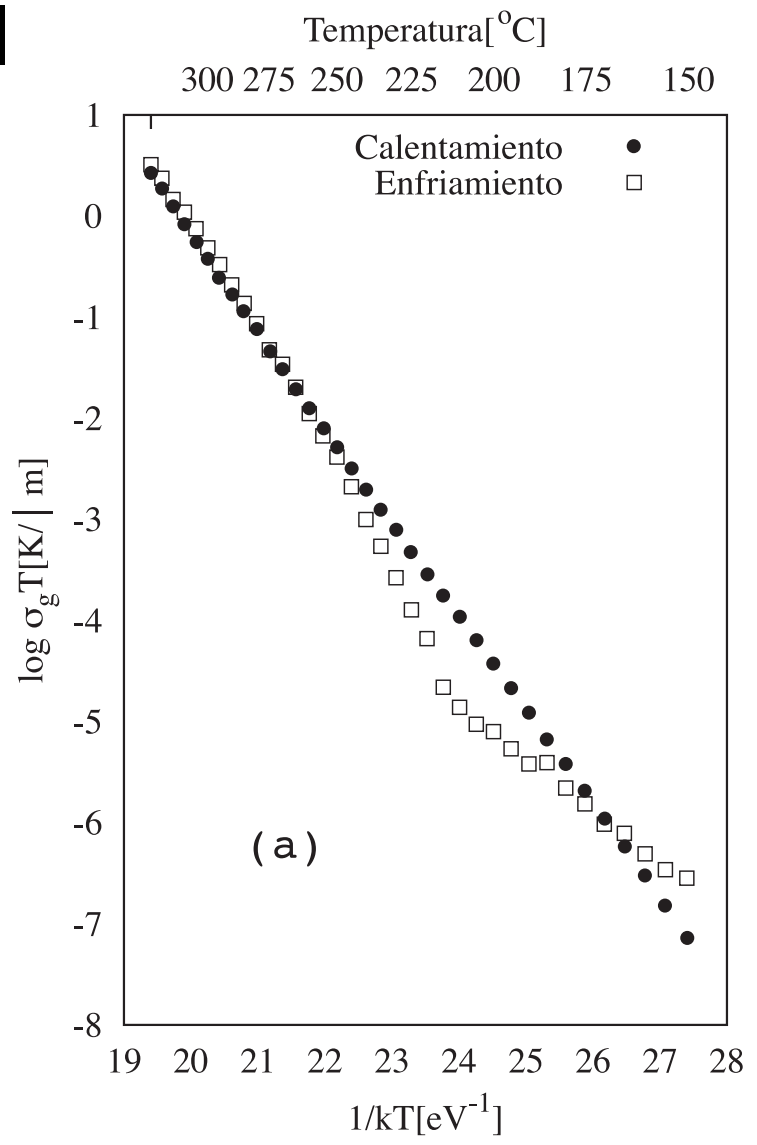

b

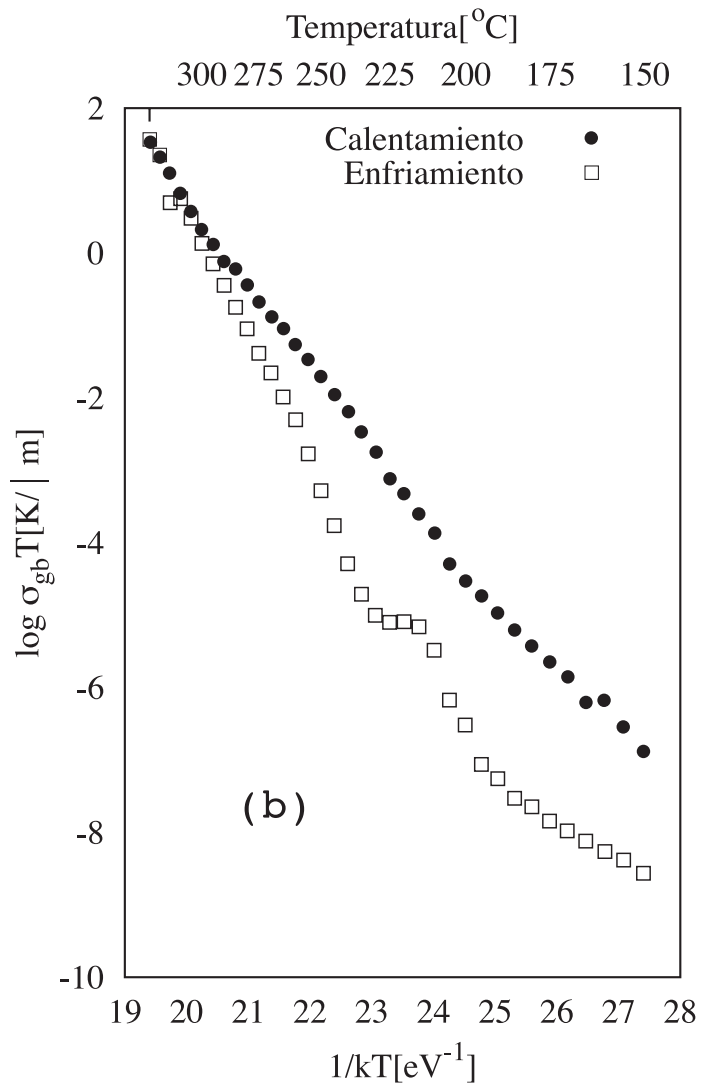

Figura 5. Diagramas de Arrhenius de la conductividad de grano (a) y frontera de grano (b) de la muestra de $0.7 \mu \mathrm{m}$ durante el ciclo 1 (calentamiento) y ciclo 2 (enfriamiento).
La principal diferencia con la muestra de $0.7 \mu \mathrm{m}$ (véase la figura 5) es la rapidez con la que se producen los cambios. Mientras que en la muestra de $1.2 \mu \mathrm{m}$ las conductividades del grano y frontera de grano caían abruptamente en el primer calentamiento, en la muestra de $0.7 \mu \mathrm{m}$ esta caída se produce de forma gradual durante el enfriamiento. Los signos de envejecimiento comienzan a verse en la frontera de grano en torno a $300^{\circ} \mathrm{C}$ mientras que en el grano se hacen evidentes a $250^{\circ} \mathrm{C}$. Este cambio más suave puede ser explicado teniendo en cuenta que existen dos efectos que cooperan entre sí; por una parte está demostrado que tamaños de grano menores tienen una barrera de nucleación mayor (4) por lo que la velocidad de transformación es menor. Además, con un tamaño de grano menor es más fácil acomodar el incremento de volumen producido por la transformación $t \rightarrow m$ que con un tamaño de grano mayor. Para ver la evolución del espectro de impedancia hemos representado en la figura 6 la parte imaginaria de éste a $250^{\circ} \mathrm{C}$ en los seis primeros ciclos térmicos. Igual que sucede en la muestra de $1.2 \mu \mathrm{m}$ (figura 4), en el segundo ciclo el mínimo entre los máximos asociados a efectos de grano y frontera de grano se suaviza. Esto es causado por el aumento de la contribución de la frontera de grano. En ciclos térmicos posteriores se pierde todo vestigio de los dos máximos que son substituidos por un comportamiento parecido al que tiene la muestra de $1.2 \mu \mathrm{m}$. Por tanto, en los diagramas de Arrhenius de la figura 5 hay que tener en cuenta que el parámetro de ajuste que estaba inicialmente asociado a la conductividad de la frontera de grano, ahora contiene también la contribución de las grietas.

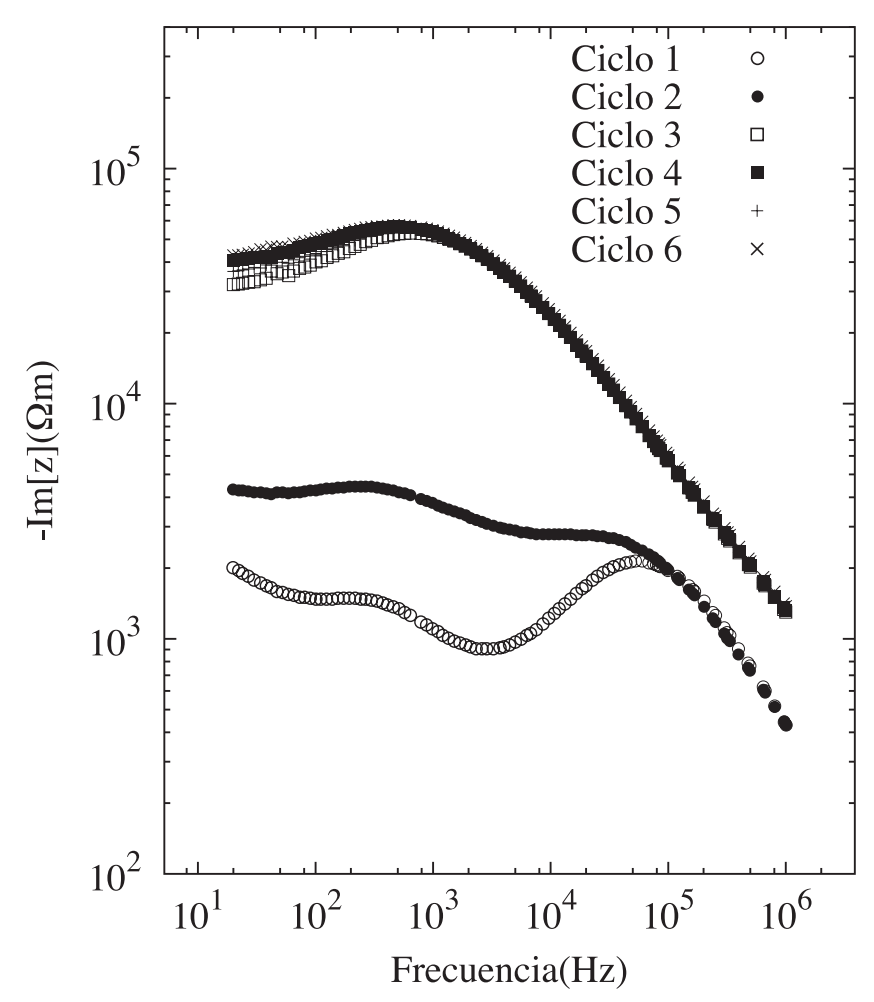

Figura 6. Parte imaginaria del espectro de impedancia de la muestra de $0.7 \mu \mathrm{m}$ a $250^{\circ} \mathrm{C}$ en los ciclos del 1 al 6. 
Finalmente, la muestra de $0.3 \mu \mathrm{m}$ permanece estable en al menos 6 ciclos térmicos ( $300 \mathrm{~h}$ aprox.), tal y como puede verse en la figura 7, en acuerdo con los resultados de Watanabe y col (1) quienes no encuentran ninguna transformación en muestras con tamaño de grano de $0.3 \mu \mathrm{m}$ después de tratamientos de $1000 \mathrm{~h}$ a $300^{\circ} \mathrm{C}$. Estos datos concuerdan con los datos de difracción de rayos X. Mediante el análisis Rietveld de los espectros se tiene que la muestra recién sinterizada está compuesta de un $(93.8 \pm 1.1) \%$ de fase tetragonal y de un $(6.2 \pm 0.6) \%$ de fase monoclínica, mientras que al final de todos los ciclos térmicos el contenido en fase tetragonal es de $(91.0 \pm 1.1) \%$ y el de monoclínica de $(9.0 \pm 0.8) \%$.

\section{CONCLUSIONES}

Se ha estudiado la respuesta eléctrica de muestras de $3 \mathrm{~mol} \%$ Y-TZP con tamaños de grano $0.3,0.7$ y $1.2 \mu \mathrm{m}$ a baja temperatura $\left(\mathrm{T}<350^{\circ} \mathrm{C}\right)$ durante varios ciclos térmicos. Se observa un proceso de envejecimiento que afecta dramáticamente a las propiedades eléctricas de las muestras; la conductividad eléctrica decrece en un factor que llega a ser de unos dos órdenes de magnitud en la muestra de mayor tamaño de grano. Se constata que el envejecimiento es más rápido en la muestra de $1.2 \mu \mathrm{m}$ que en la de $0.7 \mu \mathrm{m}$. Este hecho se relaciona con el aumento de la velocidad de transformación de fase tetragonal a monoclínica a medida que crece el tamaño de grano. El incremento de volumen de los granos monoclínicos respecto de los tetragonales origina grietas que tienen un efec-

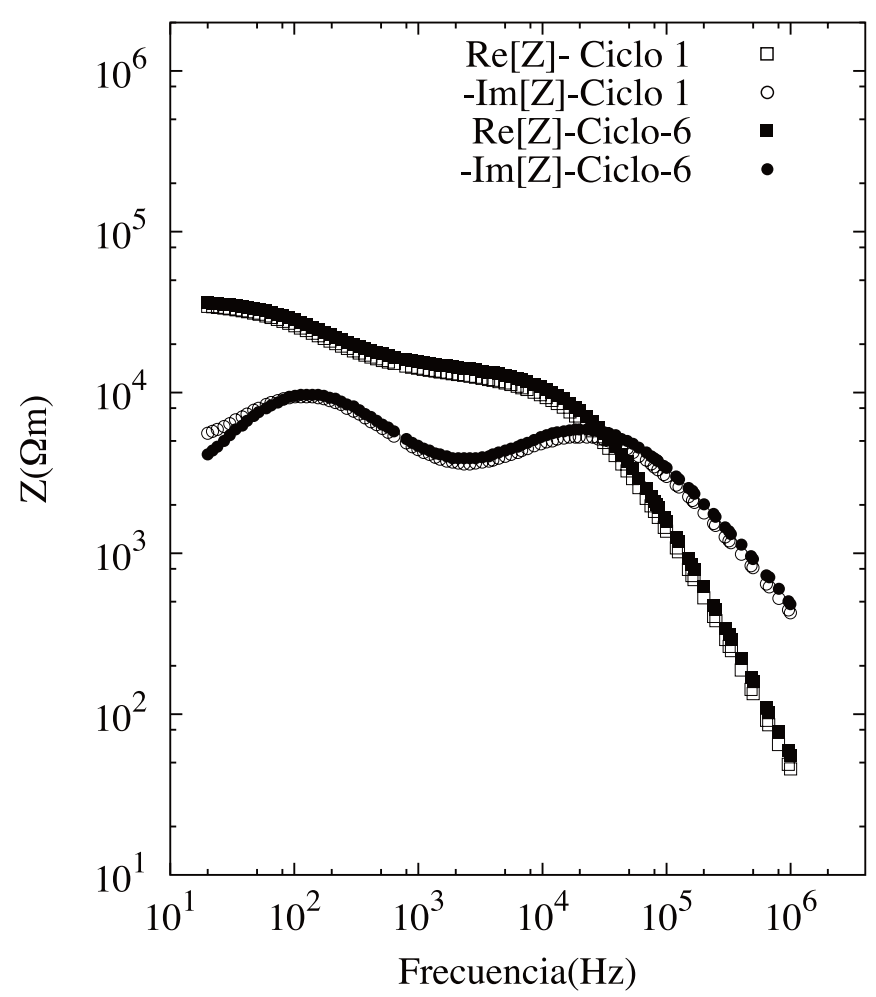

Figura 7. Espectro de impedancia de la muestra de $0.3 \mu \mathrm{m}$ en el primer y sexto ciclo térmico. to de aislante por lo que disminuye la conductividad eléctrica observada. Finalmente, la muestra de menor tamaño de grano $(0.3 \mu \mathrm{m})$ permanece estable durante al menos 600 horas.

\section{AGRADECIMIENTOS}

Este trabajo ha sido financiado por la Junta de Extremadura ( proyecto IPR00C051 y Ayudas para Estancias Breves BRV000074) y por el Ministerio de Ciencia y Tecnología MAT2000-0622.

\section{BIBLIOGRAFÍA}

1. Masakazu Watanabe, Satoshi Iio and Isamu Fukuura, "Aging behavior of Y-TZP". Advanced in Ceramics, Vol. 12, Science and Technology of Zirconia II. Editado por N. Claussen and R. Rühle and A.H. Heuer, American Ceramic Society, págs. 391-398, 1984.

2. K. Nakajima, K. Kobayashi and Y. Murata. "Phase stability of Y-PSZ in aqueous solutions". Advanced in Ceramics, Vol. 12, Science and Technology of Zirconia II, Editado por N. Claussen and R. Rühle and A.H. Heuer, American Ceramic Society, págs. 399-407, 1984.

3. K. Tsukuma, Y. Kubota and T. Tsukidate, "Termal and mechanical properties of $\mathrm{Y}_{2} \mathrm{O}_{3}$-stabilized tetragonal zirconia polycrystals". Advanced in Ceramics, Vol. 12, Science and Technology of Zirconia II, Editado por N. Claussen and R. Rühle and A.H. Heuer, American Ceramic Society, págs 382-390, 1984.

4. Jing-Feng Li and Ryuzo Watanabe. "Phase transformation in $\mathrm{Y}_{2} \mathrm{O}_{3}$-partiallystabilized $\mathrm{ZrO}_{2}$ polycrystals of various grain sizes during low-temperature aging in water". J. Am. Ceram. Soc, 81(10) 2687-2691, 1998.

5. Jérôme Chevalier, Bernard Cales and Jean Michel Drouin. "Low-temperature aging of Y-TZP ceramics". J. Am. Ceram. Soc., 82(8):2150-2154, 1999.

6. S.P.S. Badwal. "Grain boundary resistivity in zirconia-based materials: effect of sintering temperatures and impurities". Solid State Ionics, 76:67-80, 1995.

7. I. Cachadiña, J.D. Solier, F. Sánchez-Bajo y A. Domínguez-Rodríguez. "Uniones superplásticas de muestras Y-TZP: comportamiento eléctrico". En revisión, Bol. Soc. Esp. Ceram. Vidrio.

8. I. Cachadiña, J.D. Solier, A. Domínguez-Rodríguez. "Comportamiento eléctrico de monocristales 4.7 mol\% Y-PSZ". Bol. Soc. Esp. Ceram. Vidrio, 37 [23], 238-242, 1998.

9. I. Cachadiña, D. Gómez-García, J.D. Solier and A. Domínguez-Rodríguez. "Low Temperature Dielectric behaviour of yttria fully-stabilized zirconia single crystals". Radiation Effects and Defects in Solids, 151, 137-143, 1999.

10. M. Andrés-Vergés, C. Fernández-González, M. Martínez-Gallego, J.D. Solier, I.Cachadiña and E. Matijevic. "A new route for the synthesis of calcium-deficient hydroxyapatites with low $\mathrm{Ca} / \mathrm{P}$ ratio: Both spectroscopic and electric characterization". J. Mater. Res., Vol 15, No. 11, 2526-2533, 2000

11. J.R. Macdonald. "Impedance Spectroscopy". John Wiley and Sons, 1987.

12. William H. Press, Saul. A. Teukolsky, William T. Vetterling and Brian P. Flannery, "Numerical Recipes in C", Cambridge University Press, 1994

13. I. Cachadiña, J.D. Solier, F. Sánchez-Bajo y A. Domínguez-Rodríguez. "Respuesta eléctrica de nanocristales $\mathrm{ZrO}_{2}-3 \mathrm{~mol} \% \mathrm{Y}_{2} \mathrm{O}_{3}$ ". Bol Soc. Esp. Ceram. Vidrio, 38 [6],611-614 (1999)

14. A.P. Sellars and B.C.H. Steele. "The effect of $\mathrm{Y}_{2} \mathrm{O}_{3}$ on the oxygen ion conductivity of stabilized $\mathrm{ZrO}_{2}$ ". Euro-Ceramics II: Electroceramics and ceramics for special applications, Editado por G. Ziegler and H. Hausner. Deutsche Keramische Gesellschaft e.V., págs. 2091-2095, 1991.

Recibido: 26.11 .01

Aceptado: 19.12.01 